\title{
Comparative Study of Ascorbic Acid and Tocopherol Concentrations in Hydroponic- and Soil-Grown Lettuces
}

\author{
Drew N. Buchanan, Stanley T. Omaye \\ Agriculture, Nutrition and Veterinary Sciences Department, University of Nevada, Reno, USA. \\ Email: omaye@unr.edu
}

Received July $31^{\text {st }}, 2013$; revised August $31^{\text {st }}, 2013$; accepted September $7^{\text {th }}, 2013$

Copyright (C) 2013 Drew N. Buchanan, Stanley T. Omaye. This is an open access article distributed under the Creative Commons Attribution License, which permits unrestricted use, distribution, and reproduction in any medium, provided the original work is properly cited.

\begin{abstract}
Hydroponically-grown produce may be a way of helping to feed the world a variety of fruits and vegetables, regardless of soil quality, space availability or climate. The objective of this study was to determine whether hydroponicallygrown lettuce contains as much ascorbic acid and tocopherol as soil-grown lettuce. We analyzed four varieties of lettuce, including: Waldmann's Dark Green, Red Lollo Antago, Red Romaine Annapolis, and Butterleaf. The tocopherol content of hydroponically-grown Waldmann's Dark Green, Red Lollo Antago, and Red Romaine Annapolis was 229\%, $497 \%$ and $492 \%$ higher, respectively, compared to their soil-grown counterpart. The ascorbic acid content of hydroponically-grown Waldmann's Dark Green, Red Lollo Antago, and Red Romaine Annapolis was 93\%, 171\% and 216\% higher, respectively, compared to their soil-grown counterparts. Hydroponically grown lettuce varieties are significantly higher in both ascorbic acid and tocopherol content than their soil-grown counterparts, and hydroponic gardening is a viable option for producing nutritious fruits and vegetables.
\end{abstract}

Keywords: Hydroponic; Lettuce; Ascorbic Acid; Tocopherol; Leafy Vegetables

\section{Introduction}

Hydroponics offer a possible solution to a multitude of world hunger issues, from lack of farmable land to lowering transportation costs. This study was carried out to determine whether hydroponically-grown lettuce is as nutritionally dense as soil-grown lettuce, focusing on ascorbic acid and tocopherol concentrations. Few studies have been conducted to compare the nutritional quality of these two growing methods, and even fewer within the same produce species. Since the solution used to grow crops can be isolated and manipulated, the varieties of crops that can be produced are limitless.

Bioactive compounds are a fast rising topic of study. The function of flavonoids and other compounds that give plants color and protection may contribute more to health than previously thought [1]. Bioactive compounds function as antioxidants and help protect against oxidation-causing diseases, including cardiovascular disease and cancer [1]. Consumption of fruits and vegetables are associated with a healthy lifestyle, which is why it is imperative that everyone in every part of the world has access to fresh sources of produce. The National Aeronautics and Space Administration (NASA) began investigat- ing the use of hydroponics for cultivation of crops in space as far back as 1945 [2]. Not only are they useful for recycling oxygen, but provide a food source for very long space voyages [2].

The nutrients ascorbic acid and tocopherol were specifically chosen for testing because of their roles in antioxidant protection [3]. Lettuce was chosen for testing because it is one of the easier plants to grow in a greenhouse, and it was available from both commercial and greenhouse sources. The method for testing was adapted from procedures used to test the tocopherol and ascorbic acid concentrations in biological samples [4,5]. Based on other studies that compared hydroponic and soil conditions using basil [6] and lettuce [7,8], we hypothesized that hydroponically-grown lettuce would contain as much ascorbic acid and tocopherol as soil-grown lettuce, if not more.

\section{Materials and Methods}

\subsection{Varity of Lettuce}

Waldmann's Dark Green, Red Lollo Antago, and Red Romaine Annapolis lettuces were obtained from Moun- 
tain View Montessori School located in Reno, NV on May 17, 2012. All lettuces were grown at an altitude of 1398 meters (4587 feet) in a greenhouse using a static solution and aerators to provide oxygen to the roots. Fox Farm Instant Concentrated Grow Big Liquid Plant Food was diluted to create the nutrient solution. Fox Farm Soil and Fertilizer Company is located in Arcata, CA. Their nutrient solution is proprietary, and no information was found about the concentrations of each mineral contained therein. The greenhouse temperature varied from $10^{\circ} \mathrm{C}$ at night to $32^{\circ} \mathrm{C}$ during the day during the spring growing season. Nutrient solution was kept at $18^{\circ} \mathrm{C}$. A household air circulator was used to provide agitation to the leaves. The outer leaves of the lettuce heads were harvested by students of the Mountain View Montessori School. The outermost leaves were removed from the head of the lettuce using scissors.

Butterleaf lettuce from Costco (Warehouse \#25, located in Reno, NV) was purchased on May 18, 2012. The lettuce was grown hydorponically in California, USA by Indoor Farms with an Enjoy by date of May 27, 2012. The lot number was 12BL0307X. Indoor Farms determines the Enjoy by date as 15 days after harvest.

\subsection{Soil Grown Lettuce}

Waldmann's Dark Green, Red Lollo Antago, and Red Romaine Annapolis lettuces were obtained from the Mountain View Montessori School located in Reno, NV on May 17, 2012. All lettuces were grown at an altitude of 1398 meters ( 4587 feet) in the same greenhouse as the hydroponically-grown lettuces in pots of soil obtained from the school grounds (noncommercial soil enriched by natural composed material). The greenhouse temperature varied from $10^{\circ} \mathrm{C}$ at night to $32^{\circ} \mathrm{C}$ during the day. Plants were watered as the soil dried out, and soil was allowed to become dry between watering. Butterleaf lettuce from the Great Basin Community Co-op (GBCFC) was purchased on May 18, 2012. The lettuce was grown in California at Cal Organic, and distributed by Veritable Vegetable. Shipment was received at GBCFC on Tuesday of that week, just three days prior to purchase.

\subsection{Preparation of Lettuce for Analysis}

The outer leaves of lettuce were harvested by hand using scissors to separate leaves from the stem. Once leaves were harvested, they were immediately transported to the laboratory in plastic grocery bags. They were stored no longer than 1 week in a Roper RT18DKXWW00 refrigerator at $4^{\circ} \mathrm{C}$ in dark conditions between layers of paper towels in a Ziplock ${ }^{\circledR}$ plastic freezer bag. Leaves obtained from Butterleaf head lettuce plants were all separated from the core by hand and randomized before rinsing. Randomization of inner and outer leaves is important because the $\alpha$-tocopherol content of lettuce leaves vary between outer, central and inner leaves [9].

Leaves were rinsed with tap water to maximize ascorbic acid retention [10] and then spun in a commercial salad spinner to dry. One-hundred grams of lettuce was torn into approximately $1 / 2$ inch $\times 1 / 2$ inch pieces and tossed in a plastic bowl. Tearing of lettuce is a more effective method for retaining antioxidant content than cutting with a sharp knife [11]. A randomized sample of $10 \mathrm{~g}$ lettuce was selected from the bowl and used for experimentation. This is necessary due to differences in ascorbic acid content in old leaves and new leaves as well as base and tip of leaf [12]. Three $10 \mathrm{~g}$ samples were used.

\subsection{Ascorbic Acid Analysis}

Trichloroacetic acid, thiourea, copper sulfate, and sulfuric acid were purchased from Fisher Scientific, Fair Lawn, NJ. L-ascorbic acid and 2,4-dinitrophenylhydrazine were purchased from Sigma Chemical Company, St. Louis, MO.

Samples were homogenized with $10 \mathrm{~mL}$ cold $\left(7^{\circ} \mathrm{C}\right)$ 10\% Trichloroacetic acid (TCA) using a Brinkmann Instruments Polytron homogenizer to maintain a cold and acidic condition to retain ascorbic acid in the samples. Ice was utilized to maintain a cold environment when the samples were not in the centrifuge or laboratory refrigerator. Samples were then centrifuged in a Sorvall RT6000B refrigerated centrifuge for 20 minutes at 3500 $\mathrm{rpm}$ and $7^{\circ} \mathrm{C}$, then in a Sorvall Legend Micro 17 Centrifuge at $4000 \mathrm{~g}$ for 20 minutes. A stock solution of ascorbic acid was created using L-ascorbic acid and standards ranging from $0-60 \mu \mathrm{g} / \mathrm{mL}$ were created using serial dilution. One-hundred $\mu \mathrm{L}$ of supernatant was removed and added to new test tubes containing $900 \mu \mathrm{L}$ of $10 \%$ cold TCA. The colorimetric procedure first oxidized ascorbic acid to dehydroascorbic acid by reaction with copper. Addition of $0.2 \mathrm{~mL}$ dinitrophenylhydrazine (DNPH) and $0.2 \mathrm{~mL}$ thiourea in the presence of sulfuric acid to all samples, blanks, and standards yielded a colored product with little interference from other chromogens [5]. Samples were incubated for 3 hours at $37^{\circ} \mathrm{C}$ along with all of the blank and standard solutions in a Lab-Line Instruments Model 120 Incubator.

After 3 hours, $1.5 \mathrm{~mL}$ of ice cold $65 \%$ sulfuric acid was added to all solutions. After standing 30 minutes at room temperature $\left(25^{\circ} \mathrm{C}\right)$, the color stabilized and absorbances of all solutions were read at $520 \mathrm{~nm}$ in a Perkin Elmer UV/VIS lambda Bio 40 Spectrometer. A calibration curve was created from the linear regression (y $\left.=0.01668 \mathrm{x}+0.1399, \mathrm{R}^{2}>0.95\right)$ of the graph of known standards, against which samples were compared. This method measures both ascorbic acid and dehydroascorbic acid [13]. The reproducibility of spectrophotometry was 
determined by adding $300 \mu \mathrm{L}$ of a standard to a sample and determining the percent recovery [14]. Percent recovery of ascorbic acid was $136.68 \% \pm 1.31 \%$.

\subsection{Tocopherol Analysis}

4,7-Diphenyl-1,10-phanthroline (bathophenanthroline) was purchased from Aldrich, Milwaukee, WI. Ferric chloride, mixed xylenes and $( \pm) \alpha$-tocopherol were purchased from the Sigma Chemical Company, St. Louis, MO. Orthophosphoric acid (85\%) was purchased from Fisher Scientific, Fair Lawn, NJ. Absolute ethanol (200 proof, ACS/NSP grade) was purchased from pharmco-AAPER, Kentucky.

Samples were homogenized with $10 \mathrm{~mL}$ absolute ethanol using a Brinkmann instruments Polytron homogenizer. Samples were then centrifuged in a Sorvall RT6000B refrigerated centrifuge for 20 minutes at 3500 rpm and $7^{\circ} \mathrm{C}$. Samples were kept cool in the laboratory refrigerator between preparation and tocopherol assay. A stock solution of tocopherol was created using $\alpha$-tocopherol and standards ranging from $0-30 \mu \mathrm{g} / \mathrm{mL}$ were created using serial dilution. One-hundred $\mu \mathrm{L}$ of supernatant was removed and added to new test tubes containing $900 \mu \mathrm{L}$ of absolute ethanol and $1.2 \mathrm{~mL}$ of xylene to extract tocopherols from all samples, blank, and standards. After centrifugation at $3500 \mathrm{rpm}$ for 5 minutes, 0.8 $\mathrm{mL}$ of the organic layer was collected and added to new tubes containing $0.4 \mathrm{~mL}$ of bathophenanthroline. Using bathophenanthroline prevents reaction of the carotenoids with ferric chloride, which could have interfered with absorption readings. Subsequently, $0.4 \mathrm{~mL}$ ferric chloride was added, the sample was agitated using a vortex blender to promote oxidation of $\alpha$-tocopherol, and the subsequent reaction with bathophenanthroline produced an orange product. Addition of $0.4 \mathrm{~mL}$ of orthophosphoric acid stabilizes the color by preventing additional color reactions with ferric chloride. The tubes were allowed to stand for 3 minutes before absorbance measurements were taken at $536 \mathrm{~nm}$. The absorbance of each tube was read twice at $536 \mathrm{~nm}$ on a Perkin Elmer UV/VIS Lambda Bio 40 Spectrophotometer. The color was stable up to an hour and a half [15].

The concentration of tocopherol was determined using a linear regression $\left(y=0.008996 x-0.001946, R^{2}>0.95\right)$ of a curve created from standards. This method of using $\mathrm{Fe}(\mathrm{III})-$ bathophenanthroline spectrophotometry $[16]$ is slightly less reproducible when compared to HPLC methods [15], but the abundant sampling and standardization of this method allows for it to be satisfactory comparing hydroponic and soil growing conditions. The reproducibility of spectrophotometry was determined by the percent recovery method described above, with a tocopherol percent recovery of $88.74 \% \pm 2.07 \%$.

\subsection{Statistical Analysis}

Statistical analyses were conducted using Prism ${ }^{\circledR}$ for Windows, version 4.00 (Graph Pad Software, Inc., La Jolla, CA). To determine significance between varieties, one-way analysis of variance was performed using Bonferroni as a post-test when significance was observed. To determine significance between hydroponic and soil conditions, Student t-test was used. A probability value of $p$ $<0.05$ was used to determine significance. Results are expressed as mean \pm standard deviation (S.D.).

\section{Results}

Red Lollo Antago and Red Romaine Annapolis are red-leafed lettuces found to be redder in the hydroponic condition than the soil condition. Waldmann's Dark Green is a green-leafed lettuce and was found to be a darker green when hydroponically-grown than when soil-grown. No physical differences were observed between the butterleaf lettuces. Hydroponically-grown Waldmann's Dark Green, Red Lollo Antago, and Red Romaine Annapolis displayed edges of leaves that were more scalloped than the soil-grown plants.

The percent difference in tocopherol between hydroponically-grown and soil-grown Waldmann's Dark Green was $229 \%$, with the hydroponic lettuce being higher in tocopherol than the soil lettuce. Hydroponically-grown Red Romaine Annapolis was 597\% higher in tocopherol than soil-grown. Red Lollo Antago contained the highest concentration of tocopherol, with the hydroponically-grown at $492 \%$ more than soil-grown. These three varieties were found to have a significant difference in total tocopherol concentration. Soil-grown Butterleaf lettuce was found to have $45.4 \%$ less tocopherol than hydroponically-grown Butterleaf, however, this was not found to be statistically significant (Table 1).

A significant difference between the total ascorbic acid concentration of hydroponic and soil growing conditions was found in the Waldmann's Dark Green, Red Lollo Antago, and Red Romaine Annapolis varieties but not in the Butterleaf variety (Table 2). Hydroponically-grown Red Romaine Annapolis, the lettuce with the highest concentration of ascorbic acid, contained 215\% more ascorbic acid than the soil-grown Red Romaine Annapolis. Red Lollo Antago hydroponic lettuce had $171 \%$ more ascorbic acid than soil lettuce of the same variety. Waldmann's Dark Green contained 93\% more ascorbic acid in the hydroponically-grown lettuce. Butterleaf contained $183 \%$ more in the hydroponic condition, but this difference was not found to be significant (Table 2).

No significant difference in total tocopherol concentration was found between Red Lollo Antago and Red Romaine varieties of hydroponically grown lettuce. Nor 
Table 1. Total tocopherol concentration in hydroponically- and soil-grown lettuce varieties (mean \pm S.D.).

\begin{tabular}{|c|c|c|c|c|}
\hline Variety of Lettuce & $\begin{array}{l}\text { Hydroponically-grown lettuce } \\
\text { (mg/100g fresh weight) }\end{array}$ & $\begin{array}{l}\text { Soil-grown lettuce } \\
\text { (mg/100g fresh weight) }\end{array}$ & $\mathrm{t}$ & $\mathrm{p}$ \\
\hline Waldmann's Dark Green & $1.412 \pm 0.144$ & $0.428 \pm 0.230$ & 7.74 & $0.001^{*}$ \\
\hline Red Lollo Antago & $7.168 \pm 0.662$ & $1.028 \pm 0.208$ & 14.2 & $0.001^{*}$ \\
\hline Red Romaine Anapolis & $9.586 \pm 1.949$ & $1.617 \pm 0.725$ & 5.96 & $0.004^{*}$ \\
\hline Butterleaf & $0.256 \pm 0.193$ & $0.469 \pm 0.338$ & 0.86 & 0.434 \\
\hline
\end{tabular}

$\mathrm{N}=3$ with 2 replicates. ${ }^{*} \mathrm{P}<0.05$, determined using student t-test.

Table 2. Total ascorbic acid concentration in hydroponically- and soil-grown lettuce varieties (mean \pm S.D.).

\begin{tabular}{ccccc}
\hline Variety of Lettuce & $\begin{array}{c}\text { Hydroponically-Grown Lettuce } \\
(\mathrm{mg} / 100 \mathrm{~g} \text { fresh weight })\end{array}$ & $\begin{array}{c}\text { Soil-Grown Lettuce } \\
(\mathrm{mg} / 100 \mathrm{~g} \text { fresh weight })\end{array}$ & $\mathrm{t}$ & 17.6 \\
\hline Waldman's Dark Green & $3.382 \pm 0.178$ & $1.751 \pm 0.023$ & 44.5 & $0.001^{*}$ \\
Red Lollo Antago & $5.075 \pm 0.471$ & $1.872 \pm 0.035$ & 29.0 & $0.001^{*}$ \\
Red Romaine Anapolis & $7.413 \pm 0.256$ & $2.347 \pm 0.390$ & 2.63 & 0.057 \\
Butterleaf & $1.365 \pm 0.483$ & $0.482 \pm 0.141$ & & \\
\hline
\end{tabular}

$\mathrm{N}=3$ with 3 replicates. ${ }^{*} \mathrm{P}<0.05$, determined using student t-test.

was there a difference between Waldmann's Dark Green and Butterleaf of the hydroponic condition. However, there was a significant difference in total tocopherol concentration between the Red Lollo Antago and Red Romaine Annapolis versus Waldmann's Dark Green and Butterleaf (Figure 1). The former varieties contained $164 \%$ more tocopherol than the latter two varieties.

The soil-grown condition did not demonstrate any significant difference between lettuces with respect to tocopherol concentration, but significant differences in total ascorbic acid concentration were observed (Figure 2). Waldmann's Dark Green and Red Lollo Antago were significantly higher in ascorbic acid than Butterleaf, at 97\%. Red Romaine Annapolis contained 27\% more ascorbic acid than Waldmann's Dark Green and Red Lollo Antago, and 132\% more than Butterleaf.

Total ascorbic acid concentration of all hydroponically grown lettuces was found to be significantly different (Figure 3). Waldmann's Dark Green contained 85\% more ascorbic acid than Butterleaf. Red Lollo Antago contained 40\% more ascorbic acid than Waldmann's Dark Green, and Red Romaine Annapolis' ascorbic acid concentration was $37 \%$ higher than Red Lollo Antago. The hydroponically-grown lettuce with the highest total ascorbic acid concentration, Red Romaine Annapolis, contained $138 \%$ more ascorbic acid than the hydroponically-grown lettuce with the lowest ascorbic acid concentration, Butterleaf.

There were no significant differences in ascorbic acid content for soil-grown lettuce varieties (not shown).
Tocopherol Concentrations of Various Hydroponically Grown Lettuces

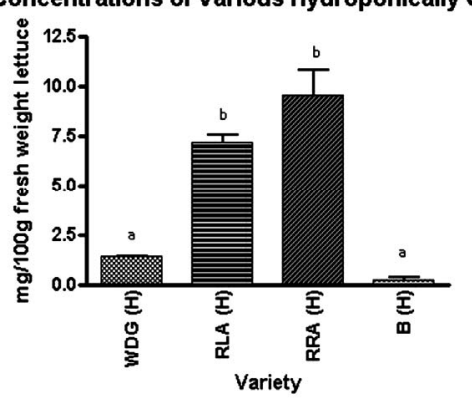

Figure 1. Tocopherol concentrations of various hydroponically grown lettuces. Lettering ab indicates significance at $\mathbf{p}$ $<\mathbf{0 . 0 5}$, with Bonferroni used as a post-test for ANOVA. Waldmann's Dark Green hydroponically grown (WDG(H)); Red Lollo Antago hydropnically grown (RLA(H)); Red Romaine Anna polis hydroponically grown (RRA(H)); Butterleaf hydroponically grown $(B(H))$.

\section{Discussion}

\subsection{Qualitative Evaluation of Lettuce}

Hydroponically grown lettuces obtained from Mountain View Montessori were slightly larger and more richly colored than their soil-grown counterparts. Waldmann's Dark Green was greener whereas the Red Lollo Antago and Red Romaine Annapolis were redder. The color differences between hydroponic lettuces and soil lettuces may be due to a difference in maturation at time of harvest. Hydroponically-grown leaves were sturdier than soil-grown leaves, the latter of which felt thin and papery. After transport, the interior of the hydroponically-grown 


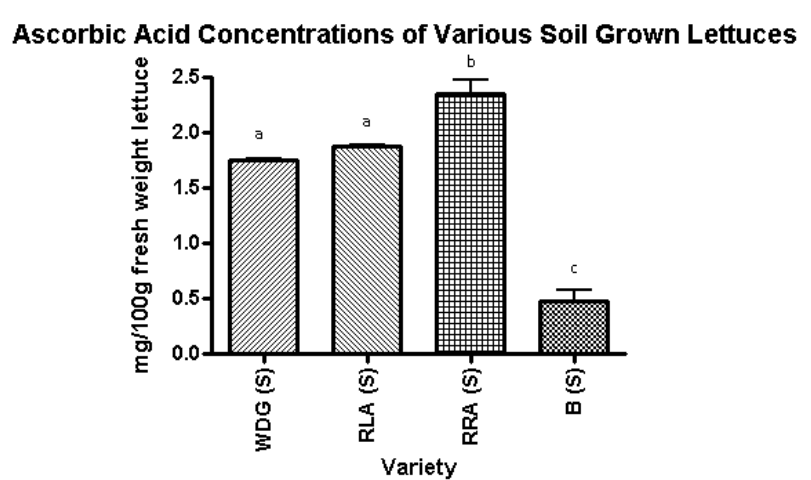

Figure 2. Ascorbic Acid concentrations of various soil grown lettuces. Lettering abc indicates significance at $\mathrm{p}<0.05$, with Bonferroni used as a post-test for ANOVA. Waldmann's Dark Green soil grown (WDG(S)); Red Lollo Antago soil grown (RLA(S)); Red Romaine Anna polis soil grown (RRA(S)); Butterleaf soil grown (B(S)).

Ascorbic Acid Concentrations of Various Hydroponically Grown Lettuces

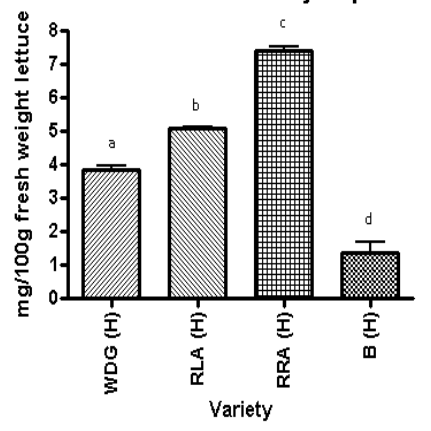

Figure 3. Ascorbic Acid concentrations of various hydroponically grown lettuces. Lettering abcd indicates significance at $p<0.05$, with Bonferroni used as a post-test for ANOVA. Waldmann's Dark Green hydroponically grown (WDG(H)); Red Lollo Antago hydropnically grown (RLA(H)); Red Romaine Anna polis hydroponically grown (RRA(H)); Butterleaf hydroponically grown $(\mathrm{B}(\mathrm{H}))$.

lettuce bags contained more condensation from the lettuce than the soil-grown lettuce bags. The hydroponically-grown and soil-grown purchased Butterleaf varieties were similar in texture and color. Indoor Farms, which produced the hydroponic Butterleaf, left the roots intact for transport and sale, which they attribute to lengthening freshness.

\subsection{Quantitative Evaluation of Lettuce}

The hydroponically-grown lettuce varieties Waldmann's Dark Green, Red Lollo Antago, and Red Romaine Annapolis contained significantly higher concentrations of tocopherol and ascorbic acid than their soil counterparts. This suggests that a hydroponic growing condition can significantly increase the nutrient density of lettuce. While there was no significant difference between varieties of soil-grown lettuces, hydroponically-grown Wald- mann's Dark Green and Butterleaf varieties showed significantly lower levels of tocopherol than hydroponically-grown Red Lollo Antago and Red Romaine Annapolis. These findings indicate that the latter two of the varieties are higher in tocopherol concentration than the former, though only when grown in hydroponic conditions.

A similar trend was seen in the Waldmann's Dark Green, Red Lollo Antago, and Red Romaine Annapolis lettuces when comparing ascorbic acid concentration in hydroponic to soil grown varieties. These three hydroponic lettuces were significantly higher in ascorbic acid than those grown in soil, with $p$ values $<0.0001$. Significant differences were seen between all varieties of hydroponic lettuce. Soil-grown Waldmann's Dark Green and Red Lollo Antago were significantly different than the other varieties. Red Romaine Annapolis displayed the highest concentrations of ascorbic acid in both hydroponic and soil grown conditions. These data suggest that the red-leafed lettuces are richer in ascorbic acid than green-leafed varieties.

This study is limited by the method used to obtain lettuces. Mountain View Montessori hydroponic lettuces were grown next to the window of the greenhouse, whereas the soil lettuces were grown on a bench on the other side of the walkway, further from the window. The circulating fan provided wind agitation to the hydroponic lettuces, but not the soil lettuces, which could be a factor in the strength of the leaves. These two aspects of growth accommodations could have contributed to the differences seen in the data collected. The composition of the soil utilized in the soil-grown condition was not analyzed for nutrient contained therein, making the comparison of the two growing methods difficult at this time. The difference in transport time between Mountain View Montessori lettuces (2 hours) and Butterleaf lettuces (5 - 6 days) makes comparison of the data more difficult. Without knowing exactly how each of the Butterleaf lettuces were transported and stored, it is difficult to recreate those conditions in the Mountain View Montessori lettuces. By growing all of the lettuce samples in a controlled laboratory setting, we believe these variables could be more tightly controlled.

Limited data exist comparing hydroponic and soil growing methods of the same lettuce varieties, but our findings are similar to what little previous research has been conducted. Sgherri et al. found that 20-day old hydroponically-grown basil was higher in both ascorbic acid and tocopherol concentration than 35-day old soilgrown basil [6]. Under our conditions, hydroponic lettuces were available for harvest earlier than the soil grown lettuces. This is consistent with the findings of Nicola et al., who found that the growth period of their hydroponic lettuces was shorter than their soil lettuces 
[17]. Since our hydroponic and soil grown lettuces were harvested on the same day, the hydroponic lettuces were further along in development than the soil lettuces, attributing to the richer red color of the hydroponic lettuces.

A study by Selma et al. compared ascorbic acid concentration in hydroponic and soil cultures of Red Lollo Rosso, Red Oak Leaf, and Butterleaf lettuces. At harvest (day 0), only Red Lollo Rosso had a significantly higher ascorbic acid concentration than soil-grown Red Lollo Rosso [8]. They found no significant difference between their Butterleaf lettuces, which is similar to what we found ( $\mathrm{p}$ value for ascorbic acid $=0.057$, $\mathrm{p}$ value for tocopherol $=0.434)$. There was no significant difference between hydroponically-grown and soil-grown Butterleaf lettuces (Tables 1 and 2). These findings are consistent with the nutritional information for soil-grown Butterleaf lettuce previously published [18] and another study comparing hydroponically- and soil-grown butterleaf lettuces [19]. Both conditions were still significantly lower in ascorbic acid and tocopherol regardless of growing method.

Before this study was conducted, few studies had been published comparing growing methods. These findings contribute more data to the comparison of hydroponically- and soil-grown lettuces. The data support the hypothesis that selected hydroponically-grown lettuce varieties are just as nutritious, if not more nutritious, than the same lettuces grown in soil when grown using Fox Farm Grow Big nutrient solution diluted according to the directions on the bottle. The nutrient solution was not manipulated to alter the ascorbic acid or tocopherol concentration in the lettuces. It is important to establish nutritional information for hydroponically-grown foods to validate the growing method.

\subsection{Influence of Growing Condition on Ascorbic Acid}

Nitrogen is an important building material for plant growth. When a hydroponic system was used to optimize a plant's access to nutrients, larger crops were obtained hydroponically compared to soil grown systems [20,21]. This phenomenon can be explained by Seginer's Nicolet Model [22], which predicts that when nitrogen and carbon uptakes are equal, there will be leaf expansion. Excess carbon, which does not occur when the nitrogen uptake is high in a hydroponic system, leads to leaf thickening. This increase in plant leaf expansion leads to a dilution effect of ascorbic acid and therefore a decrease in concentration [12].

The US Agricultural Research Station experimented in prying apart the leaves of iceberg lettuce during growth. They found that keeping such a tight leaf head from forming allowed more leaf surface to be exposed to the sun, which in turn increased the nutritional density of the plant [23]. This doubled iron and calcium and quintupled the ascorbic acid concentration of the plant. Increasing light intensity has already been shown to increase the amount of ascorbic acid a plant can instill in its leaves [11]. Ascorbic acid is a product of the conversion of sugars created during photosynthesis; increasing sunlight increases photosynthetic processes and provides more starting material to create ascorbic acid [11].

\section{Conclusion}

These data indicate that hydroponic gardening can produce a more nutrient dense lettuce than the same lettuce grown in soil. The seeds used for three of the four varieties of both hydroponic and soil growing conditions were obtained from the same distributer of seeds and grown in the same location, yet the hydroponic system produced an arguably better lettuce when considering the higher ascorbic acid and tocopherol concentrations. Variation in the concentrations of tocopherol and ascorbic acid between varieties implies that certain varieties of lettuce are more nutrient rich. We are confident in concluding that utilizing a hydroponic method of crop farming is sufficient for producing nutritious produce.

\section{Acknowledgements}

We greatly appreciate the Nevada Agricultural Experimental Station for the support of this study and lettuce donations from Cheryl Nowak and Mary Levy of the Mountain View Montessori School and statistical assistance from Fan Zhang and Dr. Wei Yang. This research was done and reported to partially fulfill thesis requirements of DN Buchanan.

\section{REFERENCES}

[1] P. M. Kris-Etherton, K. D. Hecker, A. Bonanome, S. M. Coval, A. E. Binkoski, K. F. Hilpert, et al., "Bioactive Compounds in Foods: Their Role in the Prevention of Cardiovascular Disease and Cancer," American Journal of Medicine, Vol. 113, No. 9, 2002, pp. 71-88. http://dx.doi.org/10.1016/S0002-9343(01)00995-0

[2] R. Wheeler, "Plants for Human Life Support in Space: From Myers to Mars," Gravitational and Space Biology, Vol. 23, No. 2, 2010, pp. 25-35.

[3] G. F. Combs, "The Vitamins: Fundamental Aspects in Nutrition and Health," Elsevier, Burlington, 2008.

[4] I. D. Desai and L. J. Machlin, "Vitamin E. Methods in Vitamin Assay," 4th Edition, John Wiley \& Sons WileyInterscience, New York, 1985, pp. 255-283.

[5] S. T. Omaye, J. D. Turnbull and H. E. Sauberlich, "Selected Methods for the Determination of Ascorbic Acid in Animal Cells, Tissues, and Fluids," Methods in Enzymology, Vol. 62, 1979, pp. 7-8.

http://dx.doi.org/10.1016/0076-6879(79)62181-X 
[6] C. Sgherri, S. Cecconami, C. Pinzino, F. Navari-Izzo and R. Izzo, "Levels of Antioxidants and Nutraceuticals in Basil Grown in Hydroponics and Soil," Food Chemistry, Vol. 123, No. 2, 2010, pp. 416-422.

http://dx.doi.org/10.1016/j.foodchem.2010.04.058

[7] S. Nicola, J. Hoeberechts and E. Fontana, "Comparison between Traditional and Soilless Culture Systems to Produce Rocket (Eruca sativa) with Low Nitrate Content," Acta Horticulturae, Vol. 697, 2005, pp. 549-555.

[8] M. V. Selma, M. C. Luna, A. Martínez-Sánchez, J. A. Tudela, D. Beltrán, C. Baixauli and M. I. Gil, "Sensory Quality, Bioactive Constituents and Microbiological Quality of Green and Red Fresh-Cut Lettuces (Lactuca sativa L.) Are Influenced by Soil and Soilless Agricultural Production Systems," Postharvest Biology and Technology, Vol. 63, No. 1, 2012, pp. 16-24.

http://dx.doi.org/10.1016/j.postharvbio.2011.08.002

[9] R. Szymańska and J. Kruk, "Tocopherol Content and Isomers' Composition in Selected Plant Species," Plant Physiology and Biochemistry, Vol. 46, No. 1, 2008, pp. 29-33. http://dx.doi.org/10.1016/j.plaphy.2007.10.009

[10] O. Kenny and D. O'Beirne, "The Effects of Washing Treatment on Antioxidant Retention in Ready-To-Use Iceberg Lettuce," International Journal of Food Science and Technology, Vol. 44, No. 6, 2009, pp. 1146-1156. http://dx.doi.org/10.1111/j.1365-2621.2009.01935.x

[11] S. K. Lee and A. A. Kader, "Preharvest and Postharvest Factors Influencing Vitamin C Content of Horticultural Crops," Postharvest Biology and Technology, Vol. 20, No. 3, 2000, pp. 207-220. http://dx.doi.org/10.1016/S0925-5214(00)00133-2

[12] A. Mozafar, "Decreasing the $\mathrm{NO}_{2}$ and Increasing the Vitamin C Contents in Spinach by a Nitrogen Deprivation Method," Plant Foods for Human Nutrition, Vol. 49, No. 2, 1996, pp. 155-162. http://dx.doi.org/10.1007/BF01091973

[13] M. M. Rahman, M. M. R. Khan and M. M. Hosain, "Analysis of Vitamin C (Ascorbic Acid) Contents in Various Fruits and Vegetables by UV-Spectrophotometry," Bangladesh Journal of Scientific and Industrial Research, Vol. 42, No. 4, 2007, pp. 417-424.

[14] M. M. R. Kahn, M. M. Rahman, M. S. Islam and S. A.
Begum, "A Simple UV-Spectrophotometric Method for the Determination of Vitamin C Content in Various Fruits and Vegetables at Sylhet Area in Bangladesh," Journal of Biological Sciences, Vol. 6, No. 2, 2006, pp. 388-392. http://dx.doi.org/10.3923/jbs.2006.388.392

[15] E. Tütem, R. Apak, E. Günaydi and K. Sözgen, "Spectrophotometric Determination of Vitamin E ( $\alpha$-Tocopherol) Using Copper(II)-Neocuprine Reagent," Talanta, Vol. 44, No. 2, 1997, pp. 249-255. http://dx.doi.org/10.1016/S0039-9140(96)02041-3

[16] J. Fabianek, J. DeFilippi, T. Rickards and A. Herp, "Micromethod for Tocopherol Determination in Blood Serum," Clinical Chemistry, Vol. 14, 1968, pp. 456-462.

[17] S. Nicola, J. Hoeberechts and E. Fontana, "Comparison between Traditional and Soilless Culture Systems to Produce Rocket (Eruca sativa) with Low Nitrate Content," Acta Horticulturae, Vol. 697, 2005, pp. 549-555.

[18] USDA, "Nutrient Data for 11250, Lettuce, Butterhead (Includes Boston and Bibb Types), Raw," National Agricultural Library, 2012.

Ndb.nal.usda.gov/ndb/foods/show/3067

[19] D. Frezza, A. León, V. Logegaray and A. Chiesa, "Soilless Culture Technology for High Quality Lettuce," Acta Horticulturae, No. 697, 2005, pp. 43-48.

[20] G. Coronel, M. Chang and A. Rodríguez-Delfin, "Nitrate Reductase Activity and Chlorophyll Content in Lettuce Plants Grown Hydroponically and Organically," Acta Horticulturae, No. 843, 2009, pp. 37-143.

[21] M. R. Broadley, I. Seginer, A. Burns, A. EscobarGutiérrez, I. G. Burns and P. J. White, "The Nitrogen and Nitrate Economy of Butterhead Lettuce (Lactuca savita var. capitata L.)," Journal of Experimental Botany, Vol. 54, No. 390, 2003, pp. 2081-2090. http://dx.doi.org/10.1093/jxb/erg222

[22] I. Seginer, "A Dynamic Model for Nitrogen-Stressed Lettuce," Annuls of Botany, Vol. 91, No. 6, 2003, pp. 623635. http://dx.doi.org/10.1093/aob/mcg069

[23] US-ARS, "Health-Promoting Foods: From ARS to You!" Agricultural Research Magazine, Vol. 56, 2008, Unpaginated. 